\title{
The asynchronous establishment of chromatin 3D architecture between in vitro fertilized and uniparental preimplantation pig embryos
}

Feifei $\mathrm{Li}^{1 \dagger}$, Danyang Wang ${ }^{1,2+}$, Ruigao Song ${ }^{2,3+}$, Chunwei Cao ${ }^{3,4 \dagger}$, Zhihua Zhang ${ }^{1,2^{*+}} \mathbb{D}$, Yu Wang ${ }^{3,5}$, Xiaoli $\mathrm{Li}^{1,2}$,

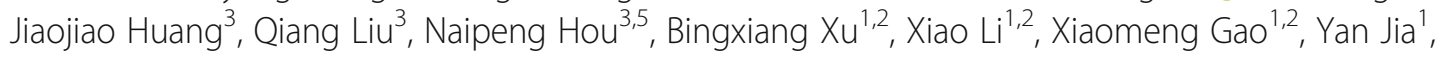
Jianguo Zhao ${ }^{2,3}$ and Yanfang Wang ${ }^{5^{*}}$

\footnotetext{
* Correspondence: zhangzhihua@ big.ac.cn; wangyanfang@caas.cn ${ }^{\dagger}$ Feifei Li, Danyang Wang, Ruigao Song, Chunwei Cao and Zhihua Zhang contributed equally to this work.

${ }^{1}$ CAS Key Laboratory of Genome Sciences and Information, Beijing Institute of Genomics, Chinese Academy of Sciences, and China National Center for Bioinformation, Beijing 100101, China

${ }^{5}$ Institute of Animal Science, Chinese Academy of Agricultural Sciences, Beijing 100193, China Full list of author information is available at the end of the article
}

\begin{abstract}
Background: Pigs are important animals for agricultural and biomedical research, and improvement is needed for use of the assisted reproductive technologies. Determining underlying mechanisms of epigenetic reprogramming in the early stage of preimplantation embryos derived from in vitro fertilization (IVF), parthenogenesis, and androgenesis will not only contribute to assisted reproductive technologies of pigs but also will shed light into early human development. However, the reprogramming of three-dimensional architecture of chromatin in this process in pigs is poorly understood.

Results: We generate three-dimensional chromatin profiles for pig somatic cells, IVF, parthenogenesis, and androgenesis preimplantation embryos. We find that the chromosomes in the pig preimplantation embryos are enriched for superdomains, which are more rare in mice. However, p(s) curves, compartments, and topologically associated domains (TADs) are largely conserved in somatic cells and are gradually established during preimplantation embryogenesis in both mammals. In the uniparental pig embryos, the establishment of chromatin architecture is highly asynchronized at all levels from IVF embryos, and a remarkably strong decompartmentalization is observed during zygotic genome activation (ZGA). Finally, chromosomes originating from oocytes always establish TADs faster than chromosomes originating from sperm, both before and during ZGA.

Conclusions: Our data highlight a potential unique 3D chromatin pattern of enriched superdomains in pig preimplantation embryos, an unusual decompartmentalization process during ZGA in the uniparental embryos, and an asynchronized TAD reprogramming between maternal and paternal genomes, implying a severe dysregulation of ZGA in the uniparental embryos in pigs.

Keywords: Pig, Early embryos, Chromatin 3D architecture, In vitro fertilization, Parthenogenesis, Androgenesis
\end{abstract}




\section{Background}

The domestic pig (Sus scrofa domesticus) is widely regarded as an important agricultural livestock species and also a crucial biomedical animal model. Given the remarkable similarity to humans, e.g., comparable size and similar early-stage embryonic development [1], the pig provides a unique model for human developmental biology research. Assisted reproductive technologies, including in vitro fertilization (IVF), parthenogenesis (PA), and androgenesis (AG), are widely used in pig reproduction and early-stage embryo development studies. Particularly, the delayed development in PA and AG embryos compared with IVF was overly severe. Thus, understanding the molecular mechanisms utilized by early stage embryos will not only contribute to increased litter size for commercial pig breeding and production, but also to the creation of genetically modified pig models for biomedical research [2]. It is well known that intensive epigenetic reprogramming and chromosome remodeling in preimplantation embryos is a prerequisite to establish a successful pregnancy [2] and that epigenetic reprogramming is reliant on the $3 \mathrm{D}$ chromatin architecture of physical bases. However, the three-dimensional structure of chromatin and its reprogramming in preimplantation development remain poorly understood for pig.

The three-dimensional (3D) architecture of the genome plays a central role in the function of nuclei and found drastic reprogramming during early embryonic development $[3,4]$. Our understanding of chromatin architecture has been deepened by chromosomal conformation capture-based methods, such as Hi-C and ChIA-PET [57]. In eukaryotic nuclei, interphase chromatin is folded in hierarchical structure, including chromosome territories (CT), compartments A and B, topologically associating domains (TADs), and chromatin loops [8-10]. Compartments A and B are associated with open and dense chromatin, respectively. TADs appear to be fundamental structural units, which are conserved across different cell types and across species. Regulatory interactions between promoter and enhancers are believed to have gone through chromatin loops. Structural proteins (e.g., CTCF and cohesin) play central roles in chromatin organization [11]. Biophysics models have been proposed, and a loop extrusion model has drawn much attention in recent years [12]. Genome-wide investigation of chromatin structure in early embryonic development has been possible with the development of low-input and single-cell Hi-C [13, 14], and multiple datasets have appeared in the literature for model systems, such as human [15], mouse [16, 17], zebrafish [18], and Drosophila [19, 20], as well as for nonmodel systems, such as medaka [21]. Overall, results obtained for all species studied indicate a reconstruction of chromatin structure. In mouse and Drosophila, severely weakened or a lack of structural features (e.g., A/B compartments or TADs) were observed before zygotic genome activation (ZGA), while structural organization emerged at the onset of or immediately after ZGA $[16,17,19]$. In a preprint paper, the authors also reported the emergence of weak structures during ZGA in medaka embryos [21]. In zebrafish, chromatin structure displays a unique systemic loss and regain pattern and the structured organizations are lost when ZGA [18]. In human, TAD is gradually established while compartments are lost in 2-cell embryos and are re-established during embryonic development [15]. The pattern and rate of the chromatin architecture establishment vary from species to species. Thus, species-specific patterns of chromatin dynamics are critical to understand characteristics of embryo development and abnormalities of special in vitro embryos. 
In this study, we generated $\mathrm{Hi}-\mathrm{C}$ maps for somatic cells and various types of preimplantation embryos (IVF, PA, and AG) in pigs. Notably, we found a potential pigspecific feature for the chromosomes in preimplantation embryos. The compartment domains that larger than $10 \mathrm{M}$ (i.e., superdomains) were much more prevalent in pig embryos than mouse embryos. Except for the superdomains, the p(s) curves, compartments, and TADs were largely conserved in somatic cells and were gradually established during preimplantation embryogenesis in both mammals. Remarkably, the establishment of chromatin architecture was highly asynchronized during the development of uniparental pig embryos, especially the strong decompartmentalization was seen during zygotic genome activation (ZGA). Our data not only provide valuable resources for evolutionary research on genome architecture, but also can be used as a unique reference for IVF and uniparental pig reproduction.

\section{Results}

Chromatin architecture is largely conserved in embryonic fibroblast cells between mouse and pig

Using pig embryonic fibroblasts (PEFs), we performed sisHi-C assay and obtained highly reproducible Hi-C maps between two replicates (Table S1 and Fig. S1A), with an average of 256 million cis-paired reads for each replicate. The reproducibility of the Hi$\mathrm{C}$ data was validated by HiCRep, GenomeDISCO, and Pearson correlation coefficient (PCC) analyses of contact matrices between replicates (Fig. S1A, B and C) [22]. The Hi-C contact map of PEF showed canonical chromatin organization, including compartments and TAD (Fig. 1a). By comparing to mouse embryonic fibroblasts (MEF), we assessed the similarity of genome architectures between pig and mouse at the chromatin contact frequency curve $\mathrm{p}(\mathrm{s}), \mathrm{A} / \mathrm{B}$ compartments, and TAD levels [23] and found that the two mammalian genome architectures were largely conserved. At the $\mathrm{p}(\mathrm{s})$ level, the similarity of two $\mathrm{p}(\mathrm{s})$ was assessed by Jensen-Shannon divergence (JSD). We found the JSD between PEFs and MEFs were 0.0035, which is comparable to the average JSD of replicates of all our samples (0.0038) (Fig. 1b). At the level of compartment, the two cell types showed similar arrangement of compartment assignments. There were $72.6 \%$ of homologous bins $(50 \mathrm{~kb})$ in PEFs that had identical compartment assignments in MEFs (Fig. 1c, see Materials and Methods), and compartment strengths between PEFs and MEFs were also comparable ( $p=0.373$, Fig. S1D). The accuracy of compartment assignments in PEFs were validated by our ChIP-seq data of two histone modification marks, H3K27ac and H3K4me3 (Table S1), and a public RNA-seq data [24]. The A compartment showed higher levels of gene expression $(p=1.84 \mathrm{E}-7$, Fig. 1d) and also showed an enrichment of H3K4me3 and H3K27ac modification marks ( $p=0.001$, Fig. 1e), compared with B compartments. At the TAD level, these two mammals also showed remarkable conservation. There were 6038 TADs identified with a median size of $360 \mathrm{~kb}$ in PEFs using our recently developed algorithm deDoc [25]. The accuracy of TAD identification was evidenced by the aggregate analysis of insulation score (Fig. 1f), and further supported by the enrichment of TSS, and CTCF binding in the TAD boundaries (Fig. 1g). Moreover, CTCF motifs within the TAD boundaries showed a clear convergent distribution (Fig. 1h), suggesting that loop extrusion may also work in pigs [8]. We found that $33.88 \%$ of pig homologous boundaries were shared in mouse 


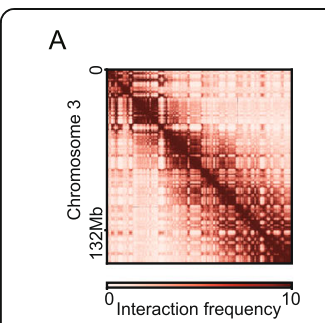

C

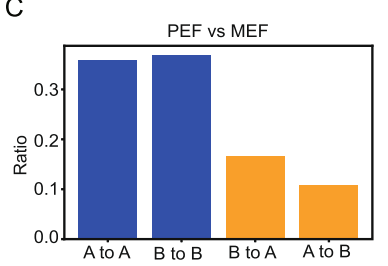

D

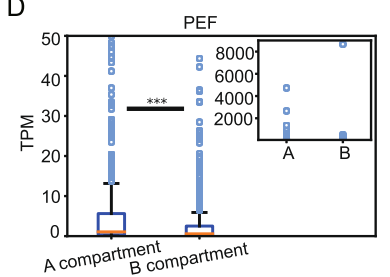

$\mathrm{H}$

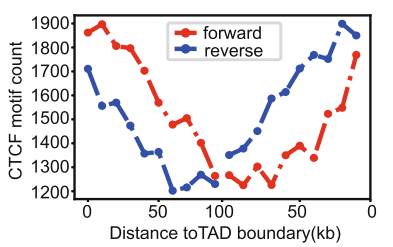

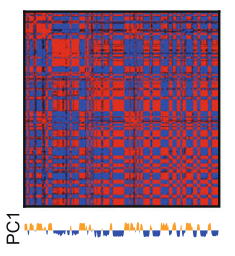

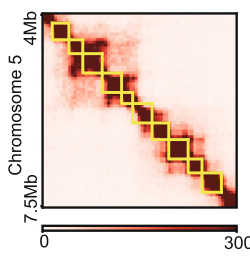

B

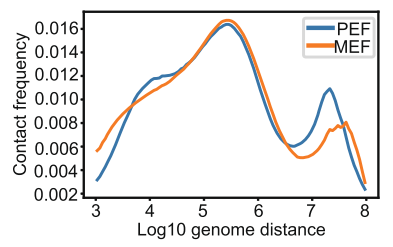

E

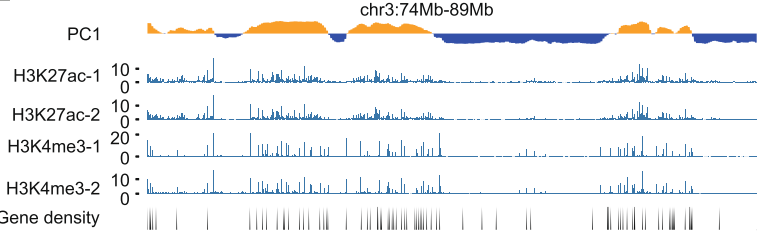

$\mathrm{F}$

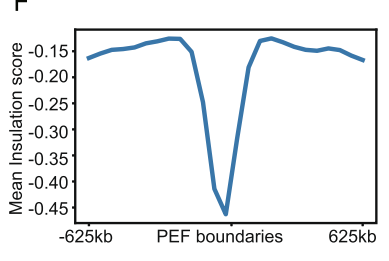

G

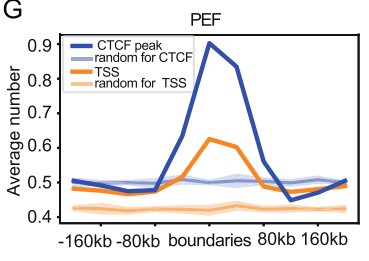

I

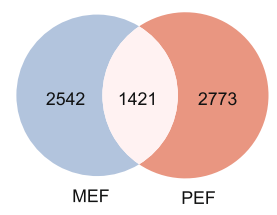

$\mathrm{J}$

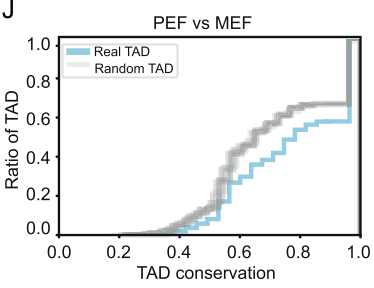

K

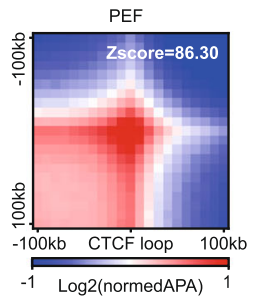

Homologous loop in MEF

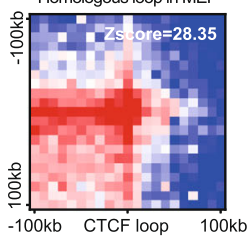

Fig. 1 Chromatin architecture of pig embryonic fibroblast. a Left: Hi-C contact matrix of chromosome 3 at 100 $\mathrm{kb}$ resolution at PEF (pooled data from 2 biological replicates). Middle: the principal component 1 (PC1) values (bottom) and correlation matrix (upper) obtained at $300 \mathrm{~kb}$ resolution for chromosome 3. Positive PC1 values represent compartment A regions (yellow), and negative values represent compartment B regions (blue). Right: TADs identified by deDoc are shown (50-kb resolution, chr5: 4-7.5 Mb). Yellow boxes represent TADs. b Relative contact frequency plot showing the percentage of contacts as a function of genome distance in PEF (blue) and MEF (orange). c Ratio of homologous bins classified by A/B compartment status between PEF and MEF. $\mathbf{d}$ Comparison of expression level between genes located in A compartments and genes located in $\mathrm{B}$ compartments of PEF ( $P=1.84 \mathrm{E}-7$, Mann-Whitney $U$ test, one-tailed). e Plots showing H3k27ac and H3k4me3 ChIP-seq signal relative to the compartments (chr3: 14-19 Mb). Gene density is also shown. $\mathbf{f}$ Mean value of insulation scores around PEF TAD boundaries (boundaries $\pm 625 \mathrm{~kb}$ ). g Average numbers of CTCF ChIP-seq peaks (blue) and gene TSS (orange) around PEF TAD boundaries (boundaries $\pm 200 \mathrm{~kb}$ ). $\mathbf{h}$ Motif count and orientation of inferred CTCF binding sites relative to PEF TAD boundaries. $\mathbf{i}$ Venn graphs showing the overlap of TAD boundaries between PEF and MEF ( $P=7.63 \mathrm{E}-67$ compared to random, Fisher's exact test). $\mathbf{j}$ Cumulative curve of TADs according to TAD conservation. TAD conservation was calculated as the probability of two homologous bins located within a TAD of PEF which also co-existence within a TAD of MEF. The same curve under shuffled TADs was shown in gray as control. $\mathbf{k}$ Aggregate loop plots showing the strength of PEF CTCF loop (left) and strength of interactions between homologous loop anchors in MEF (right) 
$(p=7.63 \mathrm{E}-67$, Fisher exact test, Fig. 1i), which is even higher than the conservation between mouse and human (18.05\% comparing MEF and IMR90, Fig. S1E). In addition, we calculated TAD conservation between MEFs and PEFs as the fraction of homologous bin pairs within a TAD in pig that is also located within one TAD in mouse. Conservation between PEFs and MEFs is about 79.29\%, which is significantly higher than controls using shuffled TAD $(72.93 \%, p=2.49 \mathrm{E}-56$ rank-sum test, Fig. $1 j$ and Fig. S1F). At the chromatin loop level, a total of 4489 CTCF loops were detected for PEFs at $10 \mathrm{~kb}$ resolution (Materials and Methods). Aggregate Peak Analysis (APA) analysis showed the enrichment of interaction between the homologous loop anchors in MEF (Fig. 1k). Together, our data showed that the pig genome is organized in compartments, TADs, and loops, and the overall architecture of chromatin is conserved between PEFs and MEFs.

\section{The prevalence of superdomains in pig preimplantation embryos}

To address whether the pig and mouse shared similar patterns in chromatin architecture reprogramming in the preimplantation embryos, we collected pig IVF, PA, and AG preimplantation embryos at the zygote, 4-cell, and morula stage, and all embryonic stages were characterized using Hoechst staining (Fig. 2a). We performed sisHi-C assay and an average of 56 million cis-paired reads were generated for each stage (Fig. 2b, Table S1). The reproducibility of $\mathrm{Hi}-\mathrm{C}$ data was validated using HiCRep, GenomeDISCO, and PCC between replicates (Fig. S1A, B and G) [22]. Here, we will discuss the dynamics of IVF embryos and later compare to PA and AG embryos. To compare developmental stages, we selected to consider zygotes, before ZGA and after ZGA stages in pig and mouse preimplantation embryos. As the major ZGA occurs in 2-cell and 4cell stages in mouse and pig, respectively, we compared zygotes, early 2-cell, and 8-cell mouse embryos with the zygotes, 4-cell, and morula pig embryos, respectively. The 8cell mouse embryo and pig morula embryo were comparable as they experienced similar numbers of cell cycles after ZGA [26]. To make the Hi-C data of different samples comparable, we downsampled the pooled valid-read pairs of each sample to the number of AG 4-cell embryos that had the lowest valid contacts.

Intriguingly, by visual inspection of the $\mathrm{PC} 1$ of $\mathrm{Hi}-\mathrm{C}$ matrices in pig preimplantation embryos (Fig. S2A), we noticed that many chromosomes were made up of only a few superdomains, where the compartment domains were larger than $10 \mathrm{Mb}$. For example, three superdomains almost covered the whole chr6 (Fig. S2A). For chr3, chr4, chr8, and chr10, the chromosome arms could even be clearly separated by the PC1 (Fig. S2A). However, such superdomains were much less prevalent in mouse preimplantation embryos. The superdomains (>10 MB) covered 54.88\%, 59.33\%, and $47.70 \%$ of the genome regions in zygote, 4-cells, and morula pig embryos, respectively, while only $28.22 \%(p=7.95 \mathrm{E}-271), 30.69 \%(P=1.17 \mathrm{E}-308)$, and $24.11 \%(p=1.32 \mathrm{E}-222)$ of the genome regions were covered by superdomains in zygotes, early 2-cell, and 8-cell mouse embryos, respectively. To quantify the prevalence of those superdomains, we plotted the accumulative curve of genome coverage as a function of domain size (Fig. 2c). The larger domains covered many more genome regions in pig preimplantation embryos than those in mouse (Fig. 2c). These superdomains in pig embryo were not caused by the sparseness of the downsampled matrices, as the identical pattern can also be seen in the raw data (Fig. S2B). Thus, the enrichment of superdomains might 
A

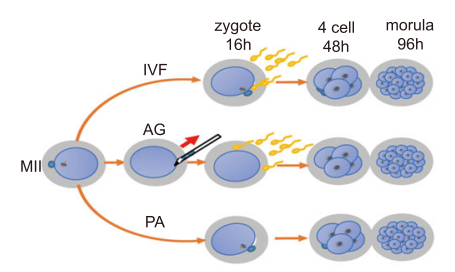

B
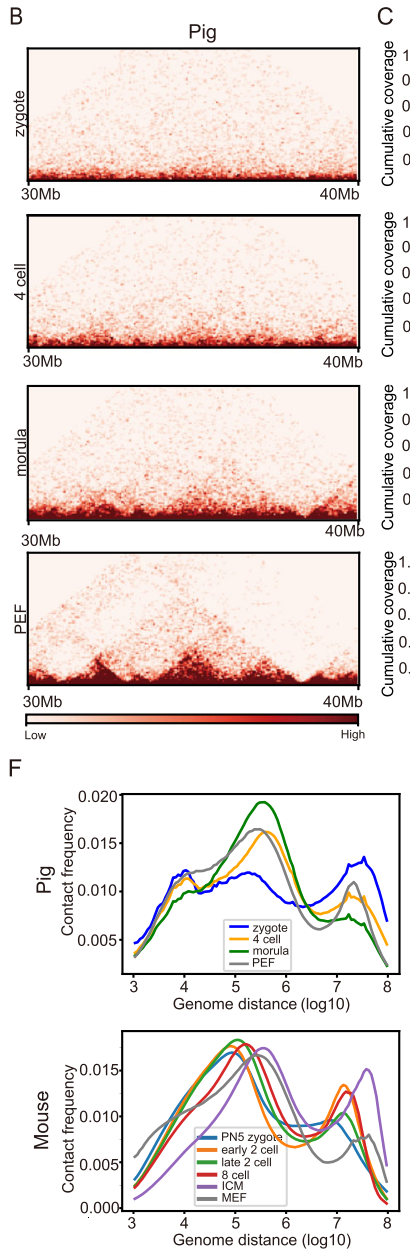

C
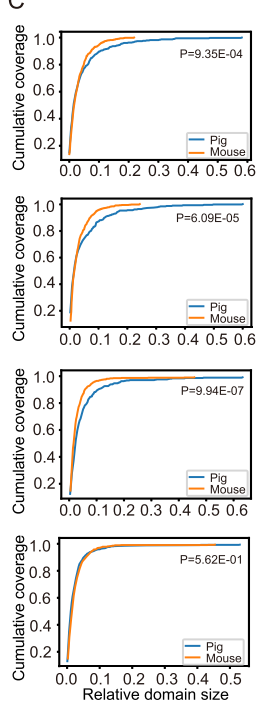

G

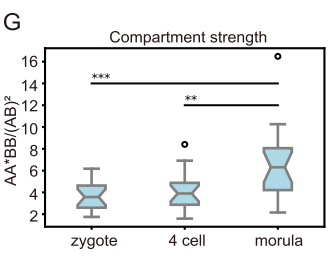

Compartment strength

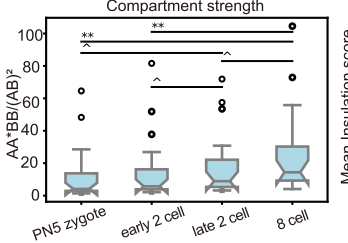

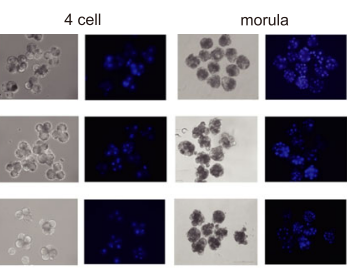

D Pig E Pig

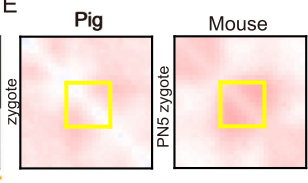

ond
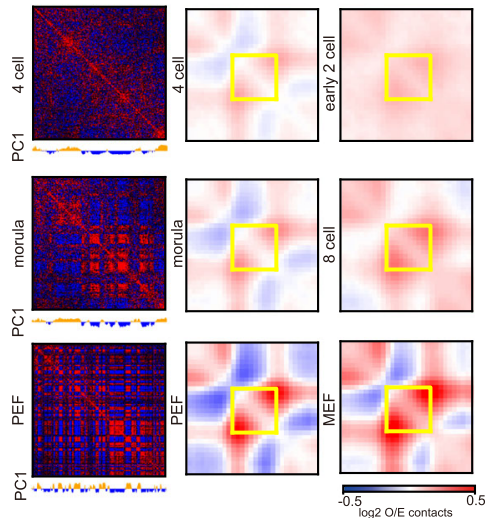

$\mathrm{H}$
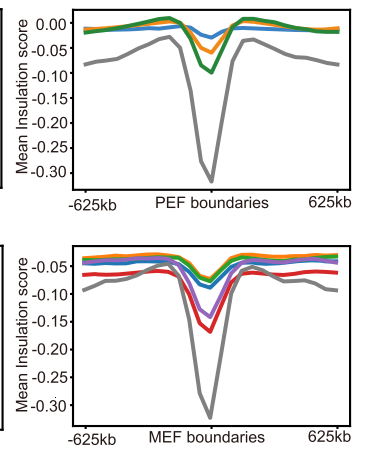

Fig. 2 Reprogramming of chromatin structure during pig in vitro fertilized early embryogenesis. a Schematic of generation of IVF, PA, and AG embryos (left) and microscopy images of 1-cell, 4-cell, and morula embryos staining with Hoechst (right). $\mathbf{b}$ Normalized $\mathrm{Hi}-\mathrm{C}$ contact heatmap of $10 \mathrm{Mb}$ region in chromosome 3 were shown at $50 \mathrm{~kb}$ resolution in the three stages of IVF embryos and PEF. c The accumulative curve for genome coverage by domains size. $X$-axis represents the relative domain size, i.e., domain length/chromosome length. Only domains larger than $1 \mathrm{MB}$ were included in this figure. The $P$ values are given for the $t$ test of average domain sizes. d PC1 and correlation matrix of chr14 at $100 \mathrm{~kb}$ resolution. e Observed/expected (O/E) aggregate plot of TADs in pig and mouse. $\mathbf{f}$ The contact frequency decay curves of Hi-C data during pig (upper) and mouse (bottom) embryogenesis. g Compartment strength which were calculated as $A A^{*} B B / A B^{2}$ for each chromosome in embryos of pig (upper) and mouse (bottom). Compartments identified in morula and 8-cell stages were used as a reference for pig and mouse, respectively. $\mathbf{h}$ Mean value of insulation scores around PEF TAD boundaries (upper) and MEF TAD boundaries (bottom). The color code is identical to f. ${ }^{*} P<0.05,{ }^{* *} P<0.01,{ }^{\wedge} P<0.1$ 
be a pig-specific feature of preimplantation embryos: however, further investigation is needed to validate this speculation.

\section{Gradual establishment of chromatin architectures during pig IVF early embryogenesis}

In general, chromatin architecture was gradually established during IVF early embryogenesis (Fig. 2b). During the process, distant contacts $(>10 \mathrm{M})$ gradually decrease, intermediate distant contacts $(>50 \mathrm{k}$ and $<1 \mathrm{M}$ ) gradually increase, and local contacts (shorter than about 20k) gradually decrease as shown by p(s) curves (Fig. 2f). Quantitatively, the JSD of $\mathrm{p}(\mathrm{s})$ curves between morula and zygotes, and between morula and 4cell embryos are 0.0227 and 0.0044 , respectively. The decreased long distant contact may indicate a gradual decompaction of chromatin, while the increased intermediate distant contacts may indicate the establishment of a megabase-sized modular structure, such as TADs (Fig. 2e). Unlike the gradual decompaction of chromatin in pig, i.e., decreased distal contact frequencies, the distal contact gradually increased in mouse during the development process (Fig. 2f).

Chromosomal segregation levels are gradually increased during pig embryogenesis, especially after ZGA (4-cell embryo, Fig. 2d and Fig. S2A). To quantify the segregation level, we identified compartments in IVF morula and took these as a reference to calculate the compartment strength $\left(\mathrm{AA}^{*} \mathrm{BB} / \mathrm{AB}^{2}\right)$ of other developmental stages. We found that compartment strength gradually increased during development, especially from 4-cell stage and morula, which showed significant enhancement (Fig. 2g). The gradually increasing segregation level could also be seen when using saddle plots to quantify compartment strength (Fig. S3A). The gradual compartmentalization could also be observed in mouse embryogenesis with a significant enhancement after ZGA (2-cell of mouse) as well (Fig. 2g and S3B). Furthermore, we found that the segregation level of the chromosomes in pig morula and mouse 8-cell embryos did not reach that in PEF and ICM, respectively (Fig. 2d), suggesting that the compartments have not be fully established at these stages [16].

At the TAD level, we found TADs were also gradually established in pig's preimplantation embryos (Fig. S4A). To quantitatively assess the establishment of TADs, we calculated the aggregate TAD signal by using the TADs identified in PEFs as the reference. Indeed, aggregate TAD signals are weak in zygotes (average $=0.530$ ) and gradually increase during development ( 0.551 and 0.563 in 4-cell stage and morula, respectively, Fig. 2e). The gradual enhancement of TADs can also be seen with decreasing insulation score (IS) at the TAD boundaries $(-0.0295,-0.0596$, and -0.0995 for the zygote, 4-cell, and morula stage, respectively, Fig. 2h). Furthermore, the overall arrangement of TADs gradually approaches the patterns seen in PEFs. Using our deDoc algorithm, we de novo identified TADs at the zygotes, 4-cell, and morula stage [25] and revealed 3822, 3347, and 3906 TADs, respectively. The TADs in PEFs were substantially similar to those in morula and less similar to the TADs in the 4-cell and zygote stage (Fig. S4B). However, both aggregate TAD signal and IS at the morula stage were still substantially weaker than those in PEFs ( 0.654 and -0.317 , respectively). Thus, the TAD structures are gradually established, but not fully established until the morula stage. Finally, for the TAD, the aggregate TAD signals also suggest a gradual establishment in zygotes, before and after ZGA stages in mouse $(0.573,0.563$, and 0.593 , respectively, Fig. 2e). This pattern can also be observed with the IS (Fig. 2h). Thus, the pattern of compartmentalization and TAD establishment 
is similar during pig and mouse embryogenesis when aligning the developmental stages based on ZGA.

At the chromatin loop level, we found that a small fraction of CTCF chromatin loops in PEFs may be established in pig preimplantation embryos, yet few such loops in MEFs are established before the ICM stage in mouse (Fig. S5, Supplementary Text).

\section{The overall dynamics of chromatin architecture in embryos from PA and AG are distinguished from IVF}

The uniparental duplications, such as PA and AG in mammals, resulted in severe embryonic defects or lethality due to genetic asymmetries between parental genome [2, 27]; however, they provided a unique model to investigate the dynamics of uniparental chromosomes during the development of preimplantation embryos. Unlike the gradual development process of IVF preimplantation embryos, both PA and AG embryos experience substantial global chromatin architecture alterations. A clear trend can be seen in the Hi$\mathrm{C}$ heatmap where the majority of the $\mathrm{Hi}-\mathrm{C}$ reads are concentrated in the narrow range near the diagonal with both of replicates showed similar (Fig. 3a, Fig. S6). This trend is also shown in the $\mathrm{p}(\mathrm{s})$ analysis (Fig. $3 \mathrm{~b}$ ), where remarkably higher peaks of local contact (shorter than about 20k) are observed in 4-cell and morula stages of PA and AG and in zygotes of PA preimplantation embryos compared with IVF embryos. Intriguingly, no local contact peaks are seen in the mouse allelic p(s) curves (Fig. 3c). In addition to the local contact, the progress of distal $(>10 \mathrm{M})$ and intermediate contacts $(<1 \mathrm{M}$ and $>50 \mathrm{~K})$ are also significantly different in more than half of the samples when comparing uniparental embryos to IVF embryos (Table S2). In PA embryos, the distal contacts are depleted in zygotes while they are drastically accumulated at the ZGA stage (4-cells), finally dropping back to mid-level at morula stages, which is equivalent to that seen at IVF morula stages (Fig. 3b). The intermediate contacts are enriched in zygotes, depleted at the 4-cell stage, and accumulated again at the morula stage. In AG embryos, however, the developmental trajectory is the opposite of that in PA embryos for the distal contact frequency, which first decreases at the 4-cell stage and then accumulates at the morula stage, whereas the trajectory for intermediate contacts is similar to that in PA. These drastic alterations of contact frequencies have not been seen in mouse allelic chromatin (Fig. 3c); thus, these differences in PA and AG chromatin architecture may reflect the abnormal nature of those uniparental embryos.

We wondered if these potentially abnormal dynamics of chromatin architecture during the development of PA and AG preimplantation embryos could be associated with the deficient inter-chromosomal contacts between the homologous chromosomes, especially since a considerable portion of collected uniparental embryos might be in fact haploid [27]. To examine this possibility, we performed a meta-analysis on inter-homologouschromosomal Hi-C reads to calculate the frequency of the homologous contacts during mouse embryogenesis [16]. We found limited homologous contacts in zygotes; however, the number of contacts sharply increased from the zygote to the late 2-cell stage and finally to the 8-cell stage, while they slightly decreased at the ICM stage (Fig. 3d). Since the chromatin architecture also emerged at the onset of ZGA in mouse, we wondered whether these homologous interactions are associated with the establishment of chromatin spatial features. To determine this possibility, we plotted the accumulative distribution 


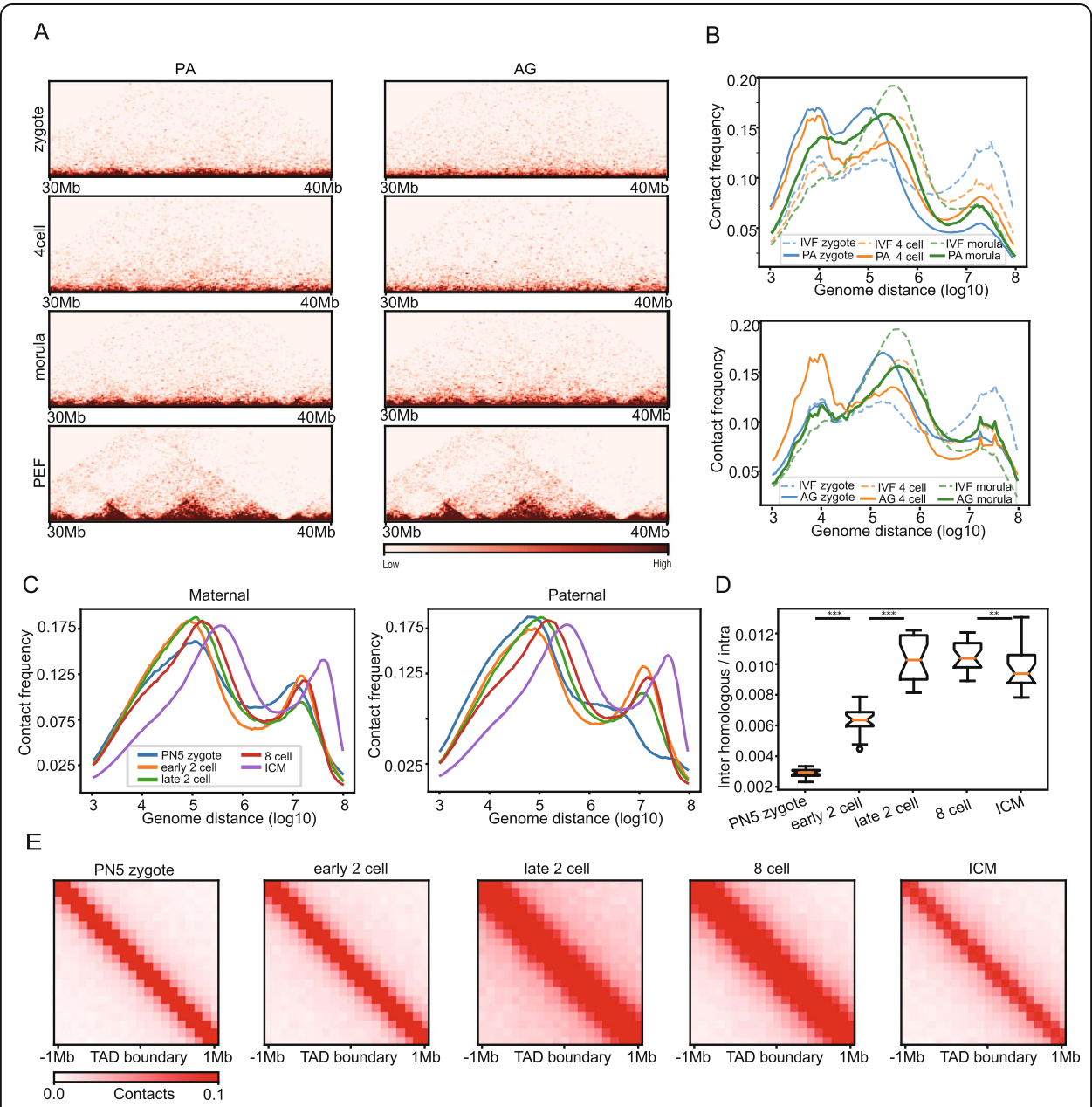

Fig. 3 The overall dynamics of chromatin architecture in embryos from PA and AG are distinguished from IVF. a Normalized Hi-C contact heatmaps for the same $10 \mathrm{Mb}$ region as Fig. 2b were shown in three stages of PA (left) and AG embryos (right). $\mathbf{b}$ The contact frequency decay curves of pig $\mathrm{Hi}-\mathrm{C}$ data during PA (upper) and AG (bottom) embryogenesis. The curves for IVF embryos are also shown. $\mathbf{c}$ The contact frequency decay curves of mouse Hi-C data in maternal (left) and paternal (right) alleles. $\mathbf{d}$ Ratio of contacts between inter-homologouschromosome and contacts of intra-chromosome for each chromosome during mouse embryogenesis. $\mathbf{e}$ Aggregate plot showing the distribution of inter-homologous-chromosome interactions (100-kb resolution) at MEF TAD boundaries and nearby regions (boundaries $\pm 1 \mathrm{Mb}$ ) during mouse embryogenesis. ${ }^{* *} P<0.001$, ${ }^{* *} P<0.01$

of the homologous contacts flanking the TAD boundaries. Our rational was that if the homologous contacts contribute to the establishment of chromatin architecture, it should not be evenly distributed along the chromosomes but be concentrated at certain structure features. However, these interactions were observed to be evenly distributed along the genome and were not enriched at TAD boundaries or within TADs (Fig. 3e). This data suggests that communication between the homologous chromosomes may not be directly associated with the establishment of TADs.

The development of A/B compartments in PA and AG preimplantation embryos is unique at the 4-cell stage

We compared the compartmentalization progress between PA, AG and IVF preimplantation embryos in pig and found divergence between the uniparental and IVF embryos 
(Fig. 4a and Fig. S7A). Although the compartmentalization pattern was similar between PA and AG embryos (Fig. S7B), the strength of compartmentalization were different. Using the compartments identified in IVF morula as the reference, we calculated compartment strengths $\left(\mathrm{AA} * \mathrm{BB} / \mathrm{AB}^{2}\right)$ in all samples. Visually, compartmentalization was found to be stronger in AG and PA zygotes than that in IVF zygotes (Fig. 4a and 2c). Quantitatively, the compartment strengths of AG zygotes are significantly higher than those of IVF zygotes, while PA zygotes are moderately higher than IVF zygotes (Fig. 4b). In the saddle plots, both PA and AG zygotes also showed higher segregation levels than IVF zygotes (Figs. S7C, D and S3A). However, the A/B compartment arrangements were similar between the three types of embryos at the zygote stage. PCC of PC1 between uniparental and IVF embryos at the zygote stage were shown to be 0.709 and 0.740 for PA and AG, respectively (Fig. 4c and Fig. S8). Strikingly, unlike the gradual compartmentalization in IVF embryos, we noticed a decompartmentalization process in both PA and AG embryos at the 4-cell stage (Fig. 4a and Fig. S9 for all chromosomes). The average compartment strengths were 3.566 and 2.769 in the PA and AG 4-cell stages, respectively, which were significantly lower than those in corresponding zygotes ( $p=0.065$ and 0.0006 , respectively) (Fig. 4b). In addition, this decompartmentalization process resulted in a substantial alteration of A/B compartment assignment over the whole genome, as evidenced by the drastic decrease in the PCC of the PC1 between uniparental and IVF embryos at the 4-cell stages (PCC $=0.620$ and 0.558 for PA and AG, respectively), as well as between PA and AG embryos ( $\mathrm{PCC}=0.563$, Fig. $4 \mathrm{c}$ and Fig. S8). At the morula stage, the compartmentalization was partially reestablished in

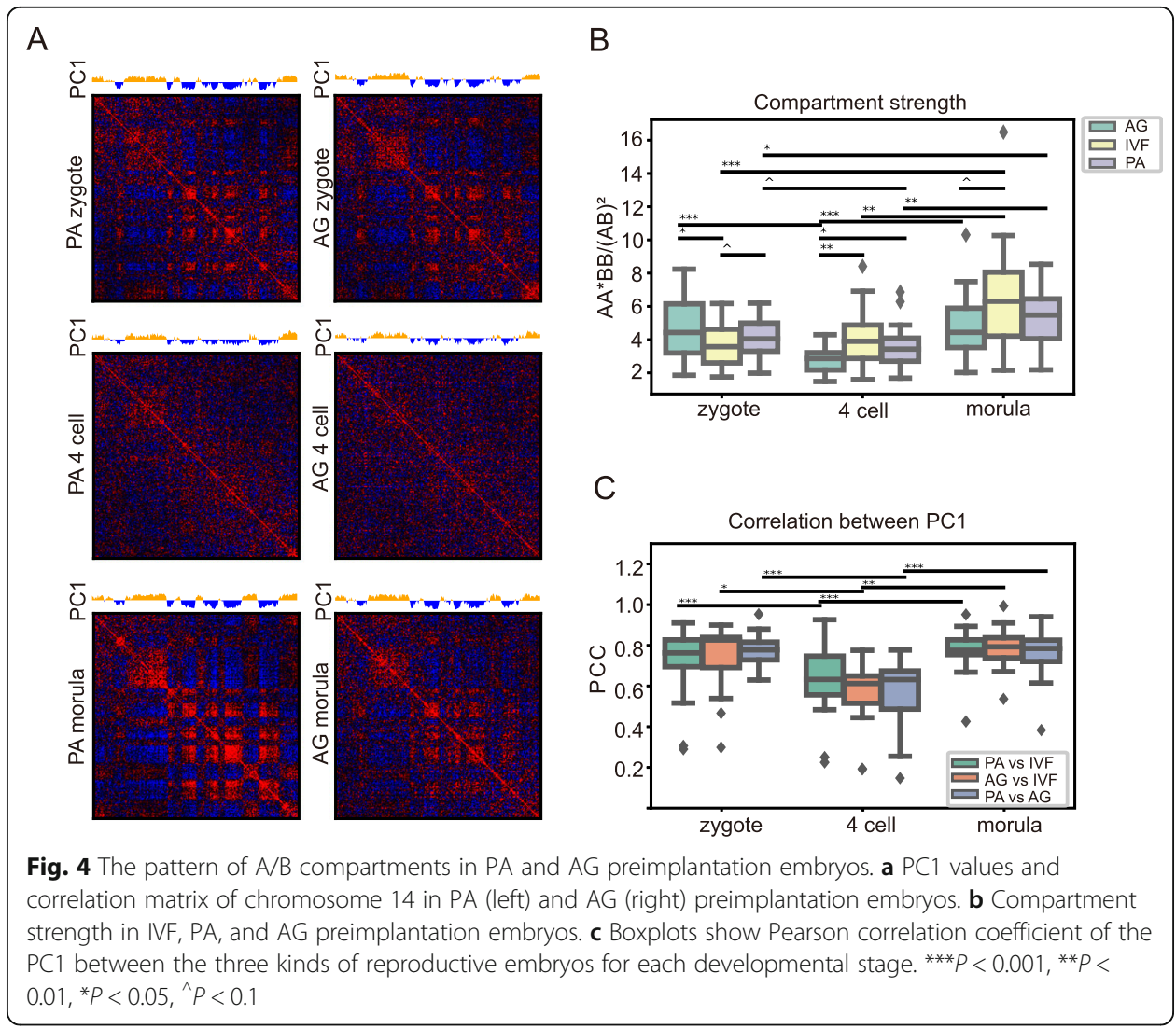


PA and AG embryos, but the degree of compartment establishment in AG was weaker than PA, while the degree was similar between PA and IVF morula (Fig. 4a and 2c). Weaker compartmentalization in AG morula was also evidenced by the average compartment strengths, showing 6.589, 5.306, and 4.934 for IVF, PA, and AG morula, respectively ( $p=0.055$ comparing AG morula with IVF morula, Fig. 4b). Furthermore, the partial reestablishment make the compartment strength of AG morula is comparable with AG zygote $(p=0.385)$. Moreover, at the morula stage, compartment reestablishment in uniparental embryos resulted in a higher similarity of PC1 with IVF morula (Fig. 4c and Fig. S8). The decompartmentalization of PA and AG at the 4-cell stage and the relatively weaker compartmentalization of AG morula can also been seen in the saddle plots (Fig. S7C and D). Together, these data indicate that the compartmental structure degrades at 4-cell stages and is reestablished at morula stages for both PA and AG preimplantation embryos, but the reestablishment is weaker in AG morula.

TADs are asynchronously established in PA and AG embryos

Because TAD structures were gradually established in IVF preimplantation embryos and gradually resembled TADs pattern of PEFs, we wondered if this might also be the case for uniparental embryos. Indeed, there was a clear trend of gradually establishing TAD boundaries in both PA and AG embryos when using PEF TAD as a reference (Fig. 5a, b and S10A). This gradual establishment of TAD boundaries was also characterized in de novo identified TADs using our deDoc algorithm (Fig. 5c). However, TAD establishment was slower in AG than in PA, as the absolute ISs of the TAD boundaries were always smaller in AG than in PA at each stage (Fig. 5a, b and S10A). Between the AG and IVF, the absolute ISs were almost identical at the zygote stage, whereas they were much smaller in AG than in IVF at the 4-cell and morula stages (Fig. 5b). Thus, TADs are gradually established and the process is much slower in preimplantation AG embryos compared with PA embryos.

Next, we asked if the asynchronous establishment of TADs in PA and AG preimplantation embryos could also been seen in the paternal and maternal chromosomes in mouse. Similar to uniparental pig embryos, the progress of TAD establishment is faster in mouse maternal chromosomes than those in paternal chromosomes at zygote, early 2-cell, and late 2-cell stages (Fig. 5d). Unexpectedly, the average IS at TAD boundaries for the maternal 8-cell stage was substantially reduced compared to the late 2-cell stage. Nevertheless, even at the ICM stage, considerable divergence in the TAD arrangement remains between the two homologous chromosomes (Fig. S10B), although the TAD strengths are almost equal between maternal and paternal TAD boundaries. Particularly, for chromosomes 7, 14, and X, TADs show rather unique patterns (Fig. S10B). These chromosome-specific, slowly established TAD structures were not seen in the uniparental preimplantation pig embryos (Fig. 5c). Together, our data indicates that TAD establishment gradually develops in pig preimplantation embryos, not only for IVF but also for uniparental embryos. The chromosomes that originated from oocytes show a faster rate of TAD development than chromosomes that originated from sperm.

Last, we identified 303, 277, and 270 boundaries with significantly different IS in zygotes, 4-cell, and morula stages for PA embryos, respectively (Fig. S11A). The encompassed genes were found to be enriched in embryonic development-related GO terms, 


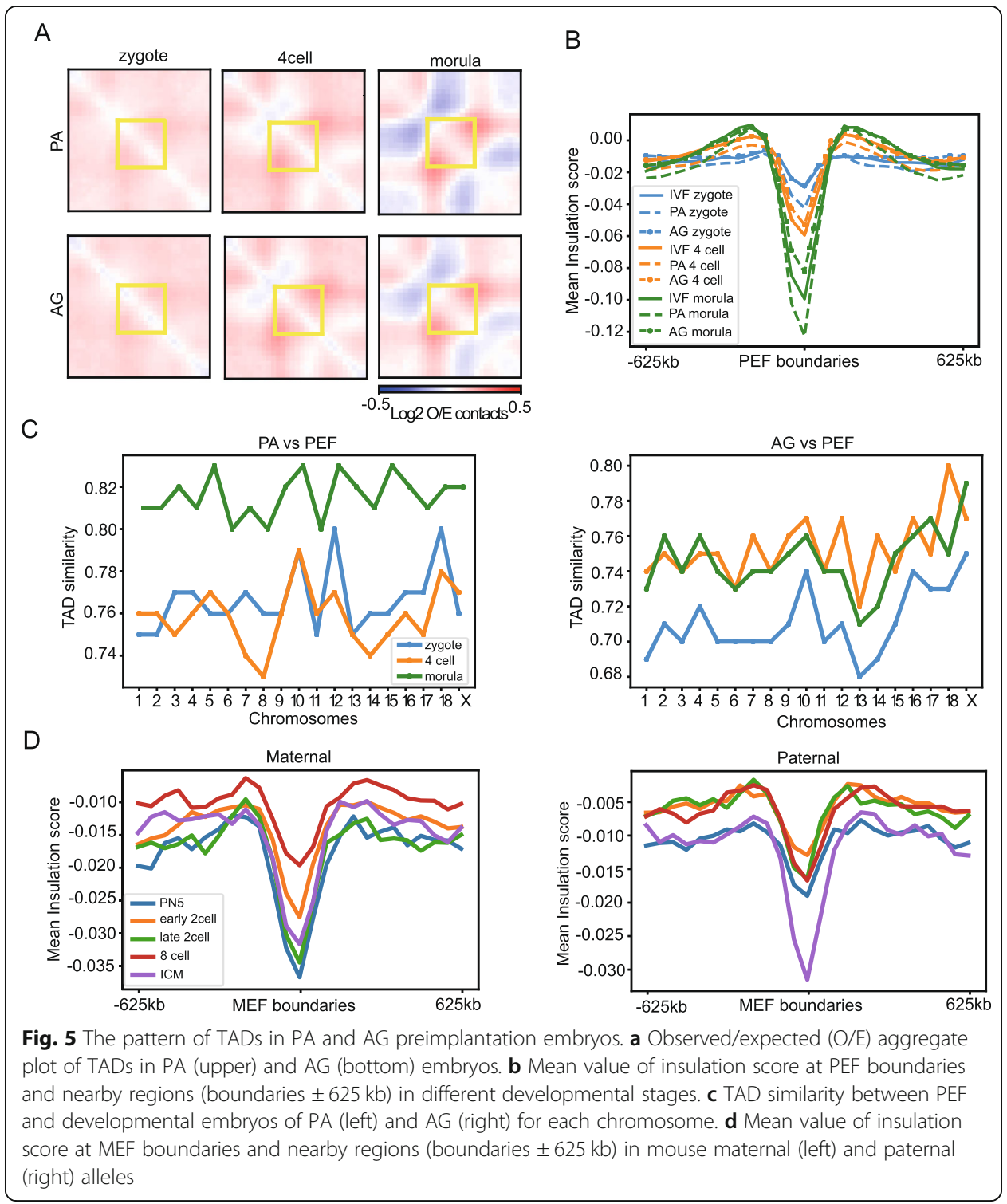

such as "embryonic organ morphogenesis" and "sperm-egg recognition" (Fig. S11B). For AG embryos, 248, 288, and 248 different boundaries were identified for zygotes, 4cell, and morula stages, respectively. And the encompassed genes were enriched in pathways associated with embryonic development and response to stimulus (Fig. S11C). Together, our results suggest that the improper establishment of chromosome architecture may be associated with abnormal activation of genes in embryogenesis, which may partially explain the decline of the in vivo developmental potential of pig uniparental embryos.

\section{Discussion}

Here, we present the first high-resolution chromosome conformation profile of pig nuclei and the dynamics of chromatin reprogramming during IVF, PA, and AG embryogenesis. The first notable finding is abnormal development at nearly all hierarchical levels of chromatin architecture in the uniparental pig embryos (Figs. 3, 4 and 5). These 
observations are striking for two reasons. First, a remarkably similar pattern of compartmentalization was found in PA and AG zygotes in the current study. In mouse, the paternal and maternal compartmentalization progress has been shown to proceed through a clear distinguished pattern and the allelic differences can be found as late as the 8-cell embryo stage [16]. For example, in the paternal genome, compartmentalization is weakly observed as early as the zygote stage; however, compartmentalization is barely observed in the maternal genome until ZGA in the late 2 -cell stage $[16,17]$. Thus, the nearly identical establishment of compartments in both PA and AG zygote embryos may imply a unique feature of pig embryo development. Particularly, the early establishment of compartments in PA zygotes should be further investigated. Second, there is an unexpected decompartmentalization process observed during ZGA at the 4-cell stage in uniparental embryos, whereas in mouse, the compartments are mainly established during ZGA [16]. Thus, these results may indicate that the compartmentalization during ZGA is critical to the reprogramming of chromatin. One possible explanation for the abnormality is that uniparental embryos contain a mixture of haploid cells as previously reported [27]. However, the abnormality may also exist in diploid embryos as haploid cells were only a minor percentage of total cells at the morula stage for both PA and AG [27]. Thus, whether this abnormality might relate to the embryonic degeneration observed in uniparental embryos is an interesting question that needs to be further investigated.

The second notable finding is the prevalence of superdomains in pig embryos and the lack in mouse, making superdomains potentially a pig-specific feature. Although further investigation is needed to validate this speculation, we would like to discuss some interesting points here. As the definition of superdomain was based on the distribution of the PC1 eigenvector, the prevalence of superdomain might represent a more outstretched arrangement of the chromosomes. It would be rather interesting to ask what caused this outstretched arrangement and determine if some pig-specific embryonic feature might contribute to this phenomenon. For example, could this phenomenon be a result of the greater number of lipid droplets present in the pig oocytes and embryos than those of other mammalian species [28]? Although the majority of lipid droplets were present in cytoplasm, there were reports that nuclear lipid droplets exist [29]. Important roles for fatty acids and lipid metabolism in the promotion of oocyte maturation and embryo development have been reported [30]. Whether the high lipid concentration plays a role in this phenomenon could be an interesting question to answer.

The third notable finding is the high concentration of local contacts of chromatin structure in pig preimplantation embryos (Figs. $2 \mathrm{f}$ and $3 \mathrm{~b}$ ), which were not enriched in mouse (Fig. 3c). This is not likely to be an artifact in the Hi-C library or an error in data processing because the peak corresponding to local contacts was much weaker in PEFs. Thus, we imagine that there might be different compositions of nanoscale chromosomal fiber $(10-30 \mathrm{~nm}$ structures [31, 32]) within pig and mouse preimplantation embryos. Obviously, this imaginative picture needs further investigation. Together, our data show that the pig genome architecture and its development in preimplantation embryos are noticeably unique.

The main motivation behind our present study is to probe a possible abnormality in chromatin architecture that could be connected to the developmental competence of pig uniparental embryos. We showed several lines of evidence to support the existence 
of such a connection. Although the chromatin structure in these uniparental embryos followed an estranged trajectory from the gradual chromatin architecture establishment process observed in IVF embryos, current data may have underestimated the abnormality of chromatin architecture in uniparental embryos and overestimated the normality in the IVF embryos. This might be accounted for by the embryos we selected to sample; our embryos are at least partially successful reprogrammed in their precursive stages and severe abnormalities carried by fatal embryos may not be present in our samples. On the other hand, although we assumed that IVF embryos were normal and could be used as a reference, the effects of in vitro fertilization and in vitro culture conditions are not negligible [33]. Further, the asynchrony we observed between the uniparental embryos did not directly indicate the existences of the asynchrony in vivo between maternal and paternal pig chromosomes. It would be invaluable if homozygote pig lines could be established in the future to address this issue. Finally, we do not have enough data or clues to even attempt to discuss what causal factors might lead to chromatin abnormal development. The early events that trigger the reprogramming of chromatin are still one of the core questions in embryo development that remains unexplored. Transcriptome [34, 35], epigenome [36], and chromatin $[16,17]$ have all been profiled in mouse preimplantation embryos and have provided events that were found to be intriguingly active at early stages, such as the activity of transposable elements [37]. However, corresponding data remains urgently needed for pig preimplantation embryos. Besides the multiple types of "omic" data, more interpretive experiments are crucial to provide causal relationships between the abnormalities we report here or other aberrations, which have yet to be identified, that contribute to the high failure rate of pig embryos.

\section{Conclusions}

We have shown the 3D chromatin structure of pig nuclei and the reprogramming of chromatin structure during embryogenesis of IVF-, PA-, and AG-derived pig embryos. Our data not only provide a valuable resource for $3 \mathrm{D}$ genome evolution research, but also a unique reference for IVF and uniparental reproduction studies. Our findings highlight that proper rate in reprogramming of chromatin architecture may play critical role in the successful development of embryos. Future studies are needed to identify the mechanistic drivers that underlie the abnormal kinetics of chromatin assembly and the establishment of 3D chromatin architecture in the early development of uniparental embryos.

\section{Methods}

\section{Collection of sperm, oocytes, and PA/IVF/AG early embryos}

Porcine follicular oocytes of Large White pigs were collected from follicles (3-6 mm in diameter) in the ovaries, which were collected from prepubertal gilts at a local slaughter house and transported to our laboratory at $\sim 38.5^{\circ} \mathrm{C}$ in saline. The cumulus-oocyte complexes (COCs) were washed three times in Hepes-buffered Tyrode medium [38] and then cultured for $42-44 \mathrm{~h}$ at $38.5^{\circ} \mathrm{C}$ under $5 \% \mathrm{CO}_{2}$ in air. The COCs were vortexed in $0.1 \%$ hyaluronidase in Hepes-buffered Tyrode medium for 4 min to eliminate the cumulus cell contaminants. Then, matured oocytes with an extruded first polar body were selected. Metaphase II (MII) stage oocytes were collected after removal of the first polar body by pronase. 
Prior to parthenogenetic activation, the matured oocytes were equilibrated for $30 \mathrm{~s}$ at $38.5^{\circ} \mathrm{C}$ in an activation medium; the oocytes were activated with two direct current pulses at $1.2 \mathrm{kV} / \mathrm{cm}$ for $30 \mu$ s provided by a BTX Electro-cell Manipulator 200 (BTX) in activation medium. Then, the activated oocytes were transferred into $500 \mu \mathrm{L}$ porcine zygote medium-3 (PZM3) and cultured at $38.5^{\circ} \mathrm{C}$ in humidified $5 \% \mathrm{CO}_{2}$. Zygotes (PN4) were collected $16 \mathrm{~h}$ after electrical stimulation, 4-cell embryos were collected 48 $\mathrm{h}$ after electrical stimulation, and the morula were collected $120 \mathrm{~h}$ after electrical stimulation. Both first and second polar bodies from embryos were removed by pronase.

In vitro fertilization (IVF) was performed as previously described [39]. Briefly, $30 \mathrm{MII}$ oocytes were transferred into $50 \mu \mathrm{L}$ droplets of modified fertilization Tris-buffered medium (mTBM) containing $10 \mathrm{mM}$ Tris, $2 \mathrm{mg} / \mathrm{ml} \mathrm{BSA}$, and $2 \mathrm{mM}$ caffeine. Frozen porcine semen in straws were thawed in $50^{\circ} \mathrm{C}$ water for $16 \mathrm{~s}$, then washed three times by centrifugation $(1900 \mathrm{~g}, 4 \mathrm{~min}$ ) with sperm wash buffer (DPBS with $1 \mathrm{mg} / \mathrm{ml} \mathrm{BSA}$ (pH 7.3)). The sperm were incubated in mTBM for $20 \mathrm{~min}$ at $38.5^{\circ} \mathrm{C}$ in $5 \% \mathrm{CO}_{2}$ humidified air. The supernatant with swimming sperm was transferred to a new well. Sperm activity was assessed in a cell counting plate before using and collecting. Fifty microliters of sperm mTBM solution was added to the fertilization droplets. The final sperm concentration was $5 \times 10^{5}$ cells $/ \mathrm{ml}$. MII oocytes were coincubated with sperm for $5 \mathrm{~h}$. After fertilization, oocytes were transferred into $500 \mu \mathrm{L}$ PZM3 medium and cultured in four-well dishes at $38.5{ }^{\circ} \mathrm{C}$ in $5 \% \mathrm{CO}_{2}$ humidified air. Zygotes (PN4), 4-cell embryos, and morula were collected $18.5 \mathrm{~h}, 48 \mathrm{~h}$, and $120 \mathrm{~h}$ after adding the sperm suspension to the fertilization droplets. All the embryos were treated with pronase to remove polar bodies, zona pellucida, and excess sperm. Finally, samples were collected from embryos, which had been confirmed by staining with $1 \mu \mathrm{g} / \mathrm{mL}$ Hoechst 33342 (Sigma).

To obtain AG embryos, the porcine oocyte enucleation and IVF was carried out as previously described [40]. Briefly, MII oocytes were enucleated through gentle aspiration of the first polar body and adjacent cytoplasm, containing chromosomes, with a beveled pipette $(17-20 \mu \mathrm{m})$ in manipulation medium supplemented with $7.5 \mathrm{mg} / \mathrm{ml}$ cytochalasin B. Enclosed cytoplasts were transferred to mTBM until IVF. After being stained with Hoechst, the presence of the maternal genome was examined under a fluorescence microscope. Completely enucleated oocytes were selected and then subjected to IVF using the above protocol. The collection procedure of the zygotes (PN4), 4-cell embryos, and morula of the AG embryos was performed as described for the IVF embryos.

\section{PEF culture and collection}

Porcine embryo fibroblast (PEF) were isolated from 35-day-old fetuses of Large White pigs, which was mechanically dissociated and digested in DMEM supplemented with $0.5 \mathrm{mg} / \mathrm{mL}$ collagenase IV for $2.5 \mathrm{~h}$ at $38^{\circ} \mathrm{C}$. Isolated PFFs were cultured in the Dulbecco's modified Eagle's medium (DMEM, HyClone) supplemented with 15\% fetal bovine serum (FBS, Gibco), $2 \mathrm{mM}$ GlutaMAX (Gibco), and 1\% nonessential amino acids (NEAA, Gibco) for $24 \mathrm{~h}$. Animal experiments were performed according to the guidelines of the Experimental Animal Welfare and Ethical of the Institute of Animal Science, Chinese Academy of Agricultural Sciences (IAS2018-11). 


\section{Preparation of sisHi-C libraries}

The sisHi-C was conducted according to previously described protocols [16, 17]. Briefly, samples were fixed with a final concentration of $1 \%$ formaldehyde and quenched with $0.125 \mathrm{M}$ glycine. Cells were lysed in ice-cold Hi-C lysis buffer $(10 \mathrm{mM}$ Tris- $\mathrm{HCl} \mathrm{pH}$ 8.0, $10 \mathrm{mM} \mathrm{NaCl}, 0.2 \%$ Igepal CA630, 1x protease inhibitor cocktail) for $15 \mathrm{~min}$. Pelleted nuclei were washed once with $1 \mathrm{x}$ NEBuffer 2 and incubated in 0.5\% sodium dodecyl sulfate (SDS) at $62{ }^{\circ} \mathrm{C}$ for $5 \mathrm{~min}$. After incubating, water and Triton X100 were added to quench the SDS. MboI restriction enzyme (NEB, R0147) was then added and chromatin was digested. Biotin-14-dATP was used to mark the DNA ends followed by proximity ligation in intact nuclei. After crosslink reversal, samples were sheared to a length of $\sim 300 \mathrm{bp}$, and then treated with the End Repair/dA-Tailing Module (NEB, E7442L) and Ligation Module (NEB, E7445L) following the manufacturer's instructions. Biotin-labeled fragments were pulled down using Dynabeads MyOne Streptavidin T1 beads (Life technologies, 65602). The Hi-C library was amplified for about 11 cycles of PCR with Q5 master mix (NEB, M0492L) following the manufacturer's instructions. DNA was then purified, quantified, and sequenced from both ends using an Illumina sequencing platform.

\section{ChIP-seq library preparation}

ChIP-seq was conducted according to previously described protocols [41] with few modifications. The cells were cross-linked with a final concentration of $1 \%$ formaldehyde followed by quenching with glycine. Cells were lysed with lysis buffer $(0.2 \%$ SDS; $10 \mathrm{mM}$ Tris-HCl, pH 8.0; $10 \mathrm{mM}$ EDTA, pH 8.0; proteinase inhibitor cocktail) and sonicated to fragments about 300-500 bp (Bioruptor, Diagenode). Dynabeads Protein A was washed twice with ChIP Buffer (10 mM Tris- $\mathrm{HCl} \mathrm{pH} 7.5,140 \mathrm{mM} \mathrm{NaCl}, 1 \mathrm{mM}$ EDTA, 0.5 mM EGTA, 1\% Triton X-100, 0.1\% SDS, 0.1\% Na-deoxycholate, Cocktail proteinase inhibitor) and was incubated with antibody (ab8580 for H3K4me3, ab4729 for $\mathrm{H} 3 \mathrm{~K} 27 \mathrm{ac}$ ) at $4{ }^{\circ} \mathrm{C}$ for $2-3 \mathrm{~h}$. The fragmented chromatin was transferred to the beadantibody complex tubes and rotated at $4{ }^{\circ} \mathrm{C}$ overnight. The beads were washed once with low salt buffer $(10 \mathrm{mM}$ Tris- $\mathrm{HCl} \mathrm{pH} 7.5,250 \mathrm{mM} \mathrm{NaCl}, 1 \mathrm{mM}$ EDTA, $0.5 \mathrm{mM}$ EGTA, 1\% Triton X-100, 0.1\% SDS, 0.1\% Na-deoxycholate, Cocktail proteinase inhibitor) and twice with high salt buffer $(10 \mathrm{mM}$ Tris- $\mathrm{HCl} \mathrm{pH} 7.5,500 \mathrm{mM} \mathrm{NaCl}, 1 \mathrm{mM}$ EDTA, 0.5 mM EGTA, 1\% Triton X-100, 0.1\% SDS, 0.1\% Na-deoxycholate, Cocktail proteinase inhibitor). After crosslink reversal, the library was constructed as $\mathrm{Hi}-\mathrm{C}$.

\section{Hi-C data processing}

The quality of all libraries was assessed using FastQC. Reads with mean quality scores less than or equal to 30 were removed. Adapters were removed by cutadapt. Pig Hi-C reads were processed using a Juicer pipeline [42]. The data was aligned against the Sscrofa11.1 reference genome. Contact reads related with ChrY and ChrMT or with $\mathrm{MAPQ}=0$ were filtered out. The correlation between $\mathrm{p}(\mathrm{s})$ was assessed with JensenShannon divergence (JSD). For each chromosome, we obtained the expected Hi-C contact values by calculating the average contact intensity for all loci at a certain distance. We then transformed the raw Hi-C matrix into an observed/expected $(\mathrm{O} / \mathrm{E})$ matrix by dividing each normalized observed value by its corresponding expected value. All 
contact matrices used for further analysis were KR-normalized with Juicer. The correlation matrixes were calculated by Juicer. To compare the contact frequencies between different samples, we randomly sampled equal numbers of valid pairs from each sample.

\section{Analysis of $A / B$ compartment}

We used HOMER software to obtain the PC1 value and A/B compartment status [43]. We used $50 \mathrm{~kb}$ and $300 \mathrm{~kb}$ resolution to obtain the PC1 value in this assay. When comparing compartments with histone markers or genes, we defined the bin size as $50 \mathrm{~kb}$. Except for PEFs, the A/B compartments were determined using the gene density of each chromosome. In PEFs, the $\mathrm{A} / \mathrm{B}$ compartments were determined using the H3K27ac ChIP-Seq signal density. The compartment strength was calculated using $\mathrm{AA} * \mathrm{BB} / \mathrm{AB}^{2}$ as previously described [44] with minor modification. To eliminate interference of interactions within TADs, we first removed local interactions shorter than 2 MB. For boxplots, we used the top $20 \%$ eigenvectors for A and the bottom $20 \%$ for B. Saddle plots were calculated as previously described with minor modifications [45]. Briefly, we first calculated $\mathrm{O} / \mathrm{E} \mathrm{Hi-C}$ matrices for $300 \mathrm{~kb}$ of binned data. Then, we sorted $\mathrm{O} / \mathrm{E} \mathrm{Hi}-\mathrm{C}$ matrix bins according to the specific $\mathrm{PC} 1$ values and divided the matrices into 50 bins. Strength of compartmentalization was defined as AA (lower right corner)/ $\mathrm{AB}$ (upper right corner) and $\mathrm{BB}$ (upper left corner)/BA (lower left corner). The values used for each corner were calculated as a mean value of 10 bins.

\section{A/B compartment conservation across species}

A/B compartment status was identified at 50-kb resolution in PEFs and MEFs. Coordinates of 50-kb bins in PEFs were converted to mm10 using the UCSC Liftover tool with default parameters [46]. Bins were considered homologous if they overlapped more than $60 \%$ with each other. Then, we calculated proportion of homologous bins classified by A/B compartment status between PEFs and MEFs.

\section{Analysis of TADs}

TADs were called using deDoc at best resolution for each chromosome [25]. ISs were calculated with bin size $50 \mathrm{~kb}$ [47]. To compare the IS between different samples, we randomly sampled equal numbers of valid pairs from each sample and calculated the IS. TAD similarity was calculated as previously described [25]. To compare TAD similarity between different stages, we used TADs called by deDoc with bin size $100 \mathrm{~kb}$. For CTCF-motifs analysis, we counted the number of CTCF-motifs in forward or reverse orientation in ten $10 \mathrm{~kb}$ bins from the TAD-boundaries. TAD strength was calculated as previously described [44]. The heatmaps of insulation score for all samples were ordered by the IS of corresponding borders in PEF. And we plotted the IS profile in a $1.25 \mathrm{Mb}$ region flanking the $\mathrm{TAD}$ borders at $50 \mathrm{~kb}$ resolution.

\section{TAD boundaries conservation across species}

For TAD comparison between pig and mouse, pig TAD boundary coordinates were converted to mm10 using the UCSC Liftover tool with default parameters [46]. Note that only domains located in syntenic regions were successfully converted; thus, only 
these domains were analyzed. After Liftover, TAD boundaries longer than $50 \mathrm{~kb}$ were discarded. Coordinates of TAD boundaries were considered similar if they overlapped with each other at least $1 \mathrm{bp}$. Randomly sampled genome loci were taken as controls.

To define conservation, we only considered the bins that could be successfully "liftover" to the mouse genome. For any given TAD in pigs, its conservation was defined as the percentage of intra-TAD bin pairs that could be liftover and the liftovered two bins also belonged to a TAD in mouse. Shuffled TADs were taken as controls for each chromosome. A bin was considered to belong to a TAD if $60 \%$ of its length overlapped with that TAD. To test the robustness of this definition, we adjusted the definition with three overlapping ratio, e.g., $0.7,0.8$, and 0.9 , and found that the conservation level was seldom effected (Fig. S1F).

\section{Convergent CTCF loop identification}

We used Fithic software to identified loops in PEFs and MEFs at $10 \mathrm{~kb}$ resolution [48]. We filtered results using a $q$ value $<0.01$ for candidate loops. The resulted loops were then filtered based on CTCF ChIP-seq peaks and CTCF direction identified by MEME [49]. We discarded loops if the distance of loop anchors were less than $300 \mathrm{~kb}$ to avoid distance decay effects. Average APA scores were calculated by Juicer with a bin size of $10 \mathrm{~kb}$.

To identify homologous CTCF loops in mouse, we converted the coordinates of both loop anchors in Sscrofa11.1 to mm10 by Liftover. Loop anchors longer than $15 \mathrm{~kb}$ were discarded. Loops were considered conserved if both converted anchors overlapped at least $1 \mathrm{bp}$ with the de novo identified loop anchors in mouse.

\section{RNA-seq data analysis}

RNA-seq data of PEFs were downloaded from GEO (SRR066366) and mapped to the pig reference genome (Sscrofa11.1) using HISAT2 with default parameters [50]. TPM values were obtained using StringTie [51].

\section{ChIP-Seq data analysis}

All ChIP-Seq reads were mapped using bowtie2 [52]. Fragments with both ends uniquely mapped with MAPQ and larger than 5 are then extracted using samtools. Duplicates were removed by Picard tools MarkDuplicates. Peaks were called using the MACS2 callpeak command with a $q$ value of 0.01 [53]. Narrow peaks were called for all libraries. Fold enrichment over control signal tracks was built using the command bdgcmp in MACS2. Peaks called from all replicates of each condition were merged according to an irreproducible discovery rate (IDR) threshold of 0.01 . Reads were then merged and a final fold enrichment over control track was made for each condition.

\section{Mouse data analysis}

Mouse embryo Hi-C data and MEF Hi-C data were downloaded from GEO (GSE82185 and GSE121087, respectively). All mouse $\mathrm{Hi}-\mathrm{C}$ reads were mapped to the mouse genome (mm10) using HiCUP pipeline v.0.5.7 [54]. We used SNPsplit software to distinguish paternal and maternal reads [55]. TADs were called by deDoc using a similar strategy as the pig data. 


\section{Supplementary information}

Supplementary information accompanies this paper at https://doi.org/10.1186/s13059-020-02095-z.

Additional file 1. Supplementary Text.

Additional file 2: Fig. S1. Reproducibility of biological replicates and conservation of chromatin architecture between PEF and MEF. Fig. S2. The prevalence of superdomains in pig preimplantation embryos. Fig. S3. Compartmentalization saddle plots of pig IVF and mouse Hi-C data. Fig. S4. Gradual establishment of TADs during pig IVF embryogenesis. Fig. S5. Loop reprogramming in pig and mouse preimplantation embryos. Fig. S6. Normalized $\mathrm{Hi}-\mathrm{C}$ contact heatmaps for the same $10 \mathrm{Mb}$ region as Fig. 3a for both replicates. Fig. S7. PC1 values and correlation matrix of chromosome 14 for both replicates and compartmentalization saddle plots of pig PA and AG $\mathrm{Hi}-\mathrm{C}$ data. Fig. S8. Pearson correlation coefficient of the PC1 using single replicates. Fig. S9. PC1 and correlation matrices of all chromosomes for pig PA and AG embryos at different stages. Fig. S10. TADs are asynchronously established in the two parental alleles. Fig. S11. Embryonic development-related genes enriched in TAD boundaries with different insulation strengths compared uniparental with IVF embryos.

Additional file 3: Table S1. Summary of Hi-C and ChIP-seq data.

Additional file 4: Table S2. Comparison of the $p(s)$ curve between uniparental embryos and the IVF embryos.

Additional file 5. Review history.

\section{Acknowledgements}

Dr. Tracey Baas and Mr. David Martin performed English language editorial services.

\section{Review history}

The review history is available as Additional file 5.

\section{Peer review information}

Anahita Bishop was the primary editor of this article and managed its editorial process and peer review in collaboration with the rest of the editorial team.

\section{Authors' contributions}

YW, ZZ, and JZ conceived this project. FL, XLL, and YJ performed the experiments, DW, BX, and XL analyzed data, RS, CC, YW, JH, QL, and NH collected the PEF and embryos, and YW, ZZ, JZ, FL, DW, CC, and XG prepared the manuscript. All authors read and approved the final manuscript.

\section{Funding}

This work was supported by the Strategic Priority Research Program of the Chinese Academy of Sciences, China (XDA24020307, XDA16030101), the National Key R\&D Program of China (2018YFC2000400), the National Transgenic Project of China (2016ZX08009003-006-007), the National Nature Science Foundation of China (31671342, 31871331, 91940304, 31672387, 81671274), and the Agricultural Science and Technology Innovation program of CAAS (ASTIPIAS05).

\section{Availability of data and materials}

The datasets generated during the current study are available in the Genome Sequence Archive in BIG Data Center (Big Data Center Members, 2017), Beijing Institute of Genomics (BIG), and Chinese Academy of Sciences, with accession numbers CRA002145 and CRA002146 (http://bigd.big.ac.cn/gsa/s/feQ4l5ET and http://bigd.big.ac.cn/gsa/s/ JxP4C43Y), and also available at NCBI's Gene Expression Omnibus (GEO) with additional processed data files under accession number GSE153452 (https://www.ncbi.nlm.nih.gov/geo/query/acc.cgi?acc=GSE153452) [56].

$\mathrm{Hi}-\mathrm{C}$ data of mouse preimplantation embryos and mouse embryonic fibroblasts (MEFs) were downloaded from the GEO database under the accession numbers of GSE82185 [16] and GSE121087 [23], respectively. RNA-seq data of PEFs were downloaded from the GEO under the accession number of SRR066366 [24].

\section{Ethics approval and consent to participate}

Animal experiments were performed according to the guidelines of the Experimental Animal Welfare and Ethical of the Institute of Animal Science, Chinese Academy of Agricultural Sciences (IAS2018-11).

\section{Consent for publication}

Not applicable.

\section{Competing interests}

The authors declare that they have no competing interests.

\section{Author details}

${ }^{1}$ CAS Key Laboratory of Genome Sciences and Information, Beijing Institute of Genomics, Chinese Academy of Sciences, and China National Center for Bioinformation, Beijing 100101, China. ${ }^{2}$ University of Chinese Academy of Sciences, Beijing 100049, China. ${ }^{3}$ State Key Laboratory of Stem Cell and Reproductive Biology, Institute of Zoology, Chinese Academy of Sciences, Beijing, China. ${ }^{4}$ Guangdong Provincial Key Laboratory of Malignant Tumor Epigenetics and Gene Regulation, Medical Research Center, Sun Yat-Sen Memorial Hospital, Sun Yat-Sen University, Guangzhou 510120, China. Institute of Animal Science, Chinese Academy of Agricultural Sciences, Beijing 100193, China. 
Received: 19 January 2020 Accepted: 7 July 2020

Published online: 10 August 2020

\section{References}

1. Kobayashi T, Zhang H, Tang WWC, Irie N, Withey S, Klisch D, Sybirna A, Dietmann S, Contreras DA, Webb R, et al. Principles of early human development and germ cell program from conserved model systems. Nature. 2017;546:41620.

2. Geisert RD, Schmitt RAM. Early embryonic survival in the pig: can it be improved? J Anim Sci. 2002;80:E54-65.

3. Bonev B, Cavalli G. Organization and function of the 3D genome. Nat Rev Genet. 2016;17:772.

4. Dekker J, Marti-Renom MA, Mirny LA. Exploring the three-dimensional organization of genomes: interpreting chromatin interaction data. Nat Rev Genet. 2013;14:390-403.

5. Fullwood MJ, Liu MH, Pan YF, Liu J, Xu H, Mohamed YB, Orlov YL, Velkov S, Ho A, Mei PH, et al. An oestrogen-receptoralpha-bound human chromatin interactome. Nature. 2009;462:58-64.

6. Lieberman-Aiden E, van Berkum NL, Williams L, Imakaev M, Ragoczy T, Telling A, Amit I, Lajoie BR, Sabo PJ, Dorschner $\mathrm{MO}$, et al. Comprehensive mapping of long-range interactions reveals folding principles of the human genome. Science. 2009;326:289-93.

7. Schmitt AD, Hu M, Ren B. Genome-wide mapping and analysis of chromosome architecture. Nat Rev Mol Cell Biol. 2016;17:743-55.

8. Denker A, de Laat W. The second decade of 3C technologies: detailed insights into nuclear organization. Genes Dev. 2016;30:1357-82.

9. Rowley MJ, Corces VG. Organizational principles of 3D genome architecture. Nat Rev Genet. 2018;19:789-800.

10. Yu M, Ren B. The three-dimensional organization of mammalian genomes. Annu Rev Cell Dev Biol. 2017;33:265-89.

11. Lee BK, lyer VR. Genome-wide studies of CCCTC-binding factor (CTCF) and cohesin provide insight into chromatin structure and regulation. J Biol Chem. 2012;287:30906-13.

12. Fudenberg G, Imakaev M, Lu C, Goloborodko A, Abdennur N, Mirny LA. Formation of chromosomal domains by loop extrusion. Cell Rep. 2016;15:2038-49.

13. Li F, An Z, Zhang Z. The dynamic 3D genome in gametogenesis and early embryonic development. Cells. $2019 ; 8: 788$.

14. Zheng $\mathrm{H}$, Xie W. The role of $3 \mathrm{D}$ genome organization in development and cell differentiation. Nat Rev Mol Cell Biol. 2019;20:535-50.

15. Chen X, Ke Y, Wu K, Zhao H, Sun Y, Gao L, Liu Z, Zhang J, Tao W, Hou Z, et al. Key role for CTCF in establishing chromatin structure in human embryos. Nature. 2019;576:306-10.

16. Du Z, Zheng H, Huang B, Ma R, Wu J, Zhang X, He J, Xiang Y, Wang Q, Li Y, et al. Allelic reprogramming of 3D chromatin architecture during early mammalian development. Nature. 2017;547:232-5.

17. Ke Y, Xu Y, Chen X, Feng S, Liu Z, Sun Y, Yao X, Li F, Zhu W, Gao L, et al. 3D chromatin structures of mature gametes and structural reprogramming during mammalian embryogenesis. Cell. 2017;170:367-81 e320.

18. Kaaij LJT, van der Weide RH, Ketting RF, de Wit E. Systemic loss and gain of chromatin architecture throughout zebrafish development. Cell Rep. 2018;24:1-10 e14.

19. Hug CB, Grimaldi AG, Kruse K, Vaquerizas JM. Chromatin architecture emerges during zygotic genome activation independent of transcription. Cell. 2017;169:216-28 e219.

20. Ogiyama Y, Schuettengruber B, Papadopoulos GL, Chang JM, Cavalli G. Polycomb-dependent chromatin looping contributes to gene silencing during Drosophila development. Mol Cell. 2018;71:73-88 e75.

21. Nakamura R, Motai Y, Kumagai M, Nishiyama H, Durand NC, Kondo K, Kondo T, Tsukahara T, Shimada A, Aiden EL, et al. CTCF looping is established during gastrulation in medaka embryos. bioRxiv. 2018. https://doi.org/10.1101/454082.

22. Yardimci GG, Ozadam H, Sauria MEG, Ursu O, Yan KK, Yang T, Chakraborty A, Kaul A, Lajoie BR, Song F, et al. Measuring the reproducibility and quality of Hi-C data. Genome Biol. 2019;20:57.

23. Gdula MR, Nesterova TB, Pintacuda G, Godwin J, Zhan Y, Ozadam H, McClellan M, Moralli D, Krueger F, Green CM, et al. The non-canonical SMC protein SmCHD1 antagonises TAD formation and compartmentalisation on the inactive $X$ chromosome. Nat Commun. 2019;10:30.

24. Petkov SG, Marks H, Klein T, Garcia RS, Gao Y, Stunnenberg H, Hyttel P. In vitro culture and characterization of putative porcine embryonic germ cells derived from domestic breeds and Yucatan mini pig embryos at days 20-24 of gestation. Stem Cell Res. 2011;6:226-37.

25. Li A, Yin X, Xu B, Wang D, Han J, Wei Y, Deng Y, Xiong Y, Zhang Z. Decoding topologically associating domains with ultra-low resolution Hi-C data by graph structural entropy. Nat Commun. 2018;9:3265.

26. Hunter RH. Chronological and cytological details of fertilization and early embryonic development in the domestic pig Sus scrofa. Anat Rec. 1974;178:169-85.

27. Park CH, Uh KJ, Mulligan BP, Jeung EB, Hyun SH, Shin T, Ka H, Lee CK. Analysis of imprinted gene expression in normal fertilized and uniparental preimplantation porcine embryos. PLoS One. 2011;6:e22216.

28. Romek M, Gajda B, Krzysztofowicz E, Kepczynski M, Smorag Z. New technique to quantify the lipid composition of lipid droplets in porcine oocytes and pre-implantation embryos using Nile red fluorescent probe. Theriogenology. 2011;75: 42-54.

29. Soltysik K, Ohsaki Y, Tatematsu T, Cheng J, Fujimoto T. Nuclear lipid droplets derive from a lipoprotein precursor and regulate phosphatidylcholine synthesis. Nat Commun. 2019;10:473.

30. McKeegan PJ, Sturmey RG. The role of fatty acids in oocyte and early embryo development. Reprod Fertil Dev. 2011;24: 59-67.

31. Song F, Chen P, Sun D, Wang M, Dong L, Liang D, Xu RM, Zhu P, Li G. Cryo-EM study of the chromatin fiber reveals a double helix twisted by tetranucleosomal units. Science. 2014;344:376-80.

32. Collepardo-Guevara R, Schlick T. Chromatin fiber polymorphism triggered by variations of DNA linker lengths. Proc Natl Acad Sci U S A. 2014;111:8061-6.

33. Bonk AJ, Li R, Lai L, Hao Y, Liu Z, Samuel M, Fergason EA, Whitworth KM, Murphy CN, Antoniou E, Prather RS. Aberrant DNA methylation in porcine in vitro-, parthenogenetic-, and somatic cell nuclear transfer-produced blastocysts. Mol Reprod Dev. 2008;75:250-64. 
34. Wang QT, Piotrowska K, Ciemerych MA, Milenkovic L, Scott MP, Davis RW, Zernicka-Goetz M. A genome-wide study of gene activity reveals developmental signaling pathways in the preimplantation mouse embryo. Dev Cell. 2004;6:133-44.

35. Hamatani T, Carter MG, Sharov AA, Ko MS. Dynamics of global gene expression changes during mouse preimplantation development. Dev Cell. 2004;6:117-31.

36. Xu Q, Xie W. Epigenome in early mammalian development: inheritance, reprogramming and establishment. Trends Cell Biol. 2018;28:237-53.

37. Rodriguez-Terrones D, Torres-Padilla ME. Nimble and ready to mingle: transposon outbursts of early development. Trends Genet. 2018:34:806-20.

38. Huang J, Zhang H, Wang X, Dobbs KB, Yao J, Qin G, Whitworth K, Walters EM, Prather RS, Zhao J. Impairment of preimplantation porcine embryo development by histone demethylase KDM5B knockdown through disturbance of bivalent H3K4me3-H3K27me3 modifications. Biol Reprod. 2015;92:72.

39. Hao Y, Mathialagan N, Walters E, Mao J, Lai L, Becker D, Li W, Critser J, Prather RS. Osteopontin reduces polyspermy during in vitro fertilization of porcine oocytes. Biol Reprod. 2006;75:726-33.

40. Sembon S, Iwamoto M, Hashimoto M, Oishi T, Fuchimoto D, Suzuki S, Yazaki S, Onishi A. Porcine androgenetic embryos develop to fetal stage in recipient mothers. Theriogenology. 2012;78:225-31.

41. Zhu W, Xu X, Wang X, Liu J. Reprogramming histone modification patterns to coordinate gene expression in early zebrafish embryos. BMC Genomics. 2019;20:248.

42. Durand NC, Shamim MS, Machol I, Rao SS, Huntley MH, Lander ES, Aiden EL. Juicer provides a one-click system for analyzing loop-resolution Hi-C experiments. Cell Syst. 2016;3:95-8.

43. Heinz S, Benner C, Spann N, Bertolino E, Lin YC, Laslo P, Cheng JX, Murre C, Singh H, Glass CK. Simple combinations of lineage-determining transcription factors prime cis-regulatory elements required for macrophage and $\mathrm{B}$ cell identities. Mol Cell. 2010;38:576-89.

44. Flyamer IM, Gassler J, Imakaev M, Brandao HB, Ulianov SV, Abdennur N, Razin SV, Mirny LA, Tachibana-Konwalski K. Single-nucleus $\mathrm{Hi}-\mathrm{C}$ reveals unique chromatin reorganization at oocyte-to-zygote transition. Nature. 2017;544:110-4.

45. Abramo K, Valton AL, Venev SV, Ozadam H, Fox AN, Dekker J. A chromosome folding intermediate at the condensin-tocohesin transition during telophase. Nat Cell Biol. 2019;21:1393-402.

46. Kuhn RM, Haussler D, Kent WJ. The UCSC genome browser and associated tools. Brief Bioinform. 2013;14:144-61.

47. Crane E, Bian Q, McCord RP, Lajoie BR, Wheeler BS, Ralston EJ, Uzawa S, Dekker J, Meyer BJ. Condensin-driven remodelling of $X$ chromosome topology during dosage compensation. Nature. 2015;523:240-4.

48. Ay F, Bailey TL, Noble WS. Statistical confidence estimation for Hi-C data reveals regulatory chromatin contacts. Genome Res. 2014;24:999-1011.

49. Bailey TL, Boden M, Buske FA, Frith M, Grant CE, Clementi L, Ren J, Li WW, Noble WS. MEME SUITE: tools for motif discovery and searching. Nucleic Acids Res. 2009;37:W202-8.

50. Kim D, Paggi JM, Park C, Bennett C, Salzberg SL. Graph-based genome alignment and genotyping with HISAT2 and HISAT-genotype. Nat Biotechnol. 2019;37:907-15.

51. Pertea M, Pertea GM, Antonescu CM, Chang TC, Mendell JT, Salzberg SL. StringTie enables improved reconstruction of a transcriptome from RNA-seq reads. Nat Biotechnol. 2015;33:290-5.

52. Langmead B, Salzberg SL. Fast gapped-read alignment with Bowtie 2. Nat Methods. 2012;9:357-9.

53. Zhang Y, Liu T, Meyer CA, Eeckhoute J, Johnson DS, Bernstein BE, Nusbaum C, Myers RM, Brown M, Li W, Liu XS. Modelbased analysis of ChIP-Seq (MACS). Genome Biol. 2008;9:R137.

54. Wingett S, Ewels P, Furlan-Magaril M, Nagano T, Schoenfelder S, Fraser P, Andrews S. HiCUP: pipeline for mapping and processing Hi-C data. F1000Research. 2015;4:1310

55. Krueger F, Andrews SR. SNPsplit: allele-specific splitting of alignments between genomes with known SNP genotypes. F1000Research. 2016;5:1479.

56. Li F, Wang D, Song R, Cao C et al. The asynchronous establishment of chromatin 3D architecture between in vitro fertilized and uniparental preimplantation pig embryos. GSE153452. Gene Expression Omnibus. https:/www.ncbi.nlm. nih.gov/geo/query/acc.cgi?acc=GSE153452 (2020).

\section{Publisher's Note}

Springer Nature remains neutral with regard to jurisdictional claims in published maps and institutional affiliations.

Ready to submit your research? Choose BMC and benefit from:
- fast, convenient online submission
- thorough peer review by experienced researchers in your field
- rapid publication on acceptance
- support for research data, including large and complex data types
- gold Open Access which fosters wider collaboration and increased citations
- maximum visibility for your research: over 100M website views per year
At BMC, research is always in progress.
Learn more biomedcentral.com/submissions

